\title{
Fruit Nutraceuticals Seeking Clinical Evidence and Technological Exploitations
}

\section{Sami Ghnimi* and Afaf Kamal-Eldin}

Department of Food science, United Arab Emirates University, Al-Ain, UAE

In the last decades, it has increasingly been recognized that diets provide humans with more than the necessary nutritional elements including proteins, fats, carbohydrates, and the levels of minerals and vitamins necessary to combat deficiency symptoms. With increased life expectation, we are in need for an adequate diet capable of providing extra benefits in terms of e.g. energy metabolism as well as bone, cardiovascular, and mental health. The historical statement of Hippocarates "Let your food be your medicine and let your medicine be your food" is persisting and gaining more importance. In 1989, Stephen L. DeFelice coined the term nutraceuticals from the words "nutrition" and "pharmaceuticals" and later defined it to include "foods, dietary supplements and medical foods that have a health-medical benefit including the prevention and/or treatment of disease" [1]. While the terms nutraceuticals and functional foods still have no regulatory definition, it is accepted by scientists and food manufacturers to include those foods and their components that provide extra health benefits to humans. Fruits and vegetables are recognized as functional foods according to this definition.

People in the Arabic peninsula daily consume the date fruit; the sweet berry produced by the tree Phoenix dactylifera, L., family Arecaceae. The date fruit is composed of the pericarp (the exocarp or a thin layer of skin, a sweet mesocarp or flesh, and a thin papery endocarp) and one seed (kernel, pit, or pyrene).Date fruits are known for a wide range of nutritional and therapeutic effects including antioxidant, anticancer, and anti-inflammatory effects [2]. Neither the contributing factors nor the exact mechanisms responsible for these effects have been characterized but it is well known that date fruits are rich in fibers, and certain minerals, vitamins, and phenolic antioxidants [3]. Date fruits contain about $10 \%$ total dietary fiber including cellulose, arabinoxylan, glucan, pectin, and lignin. Compared to other fruits and berries, date fruits contain very high levels of phenolic antioxidants, mainly belonging to the class of condensed tannins, specifically procyanidin oligomers based on (-)-epicatechin [4]. These polymers were found to amount to $\mathrm{ca} 1.5 \%$ and representing about $95 \%$ of total phenols in ripe Tunisian date fruits [5]. It is thought that the soluble tannins are responsible for the astringent taste in the early developmental stages of date fruit and that their transformation to insoluble tannins leads toastringency decline during the transition from the Kimri to Khalal stage. Phenolic acids, aglycones and derivatives of benzoic acid (gallic, protocatechuic, $p$-hydroxybenzoic, vanillic, sinapic, and syringic acid) and cinnamic acid (caffeic, hydrocaffeic, ferulic, $p$-coumaric, and syringic acids) and flavonoid glycosides of luteolin, quercetin, and apigenin were also identified in date fruits. The intestinal absorption of oligomeric tannins like procyanidin B2, B3, and B5 are known to be null or insignificant. The high concentration of antioxidants in date fruitsmay be responsible or at least partly contributing to the antiinflammatory, antimicrobial, neuroprotective, hepatoprotective, antidiabetic, and anticarcinogenic activities [2].

It remains to be explored whether the health benefits expected from the high content of antioxidants will be compromised or not by the high content of soluble sugars in date fruits (50-60\%). The sugars in most varieties of dates, mainly soft fruit types, are dominated by the invert sugars fructose and glucose while sucrose might dominate in dry and semi-dry dates. Despite their high sugar content, soft-type date fruits are classified as a food having a relatively low glycemic index (GI), which is defined as the extent to which a certain food will elevate blood glucose levels compared to an equal amount of glucose given as reference. The GI obtained in healthy individuals for different varieties of dates (Barhi, Boma'an, Dabbas, Fardh, Khalas, Lulu) were in the range of $46-55 \%$ [6]. This controversy in sugar content as related to glycemic index has been observed in honey as well as other dried fruits like raisins. There is no mechanistically-proven explanation at present for this effect but it is thought to be related to the high contents of dietary fiber and inverted sugar in these fruits. Fructose have lower bioequivalence compared to glucose (GI for fructose $=25 \%$ and for inverted sugar $=60 \%)$. The replacement of bread with date carbohydrates was found to have better effect on the post-prandial blood glucose two hours after the meal [7]. More interestingly, date fruits were also claimed to have anti-diabetic effects and diosmetin glycosides, isolated from the epicarp of date fruits, was proven to have an anti-diabetic effect on alloxan-induced diabetes in male rats [8].

The evidence for the aforementioned health benefits is scattered and lacks support from clinical and mechanistic studies. The therapeutic efficacy of mixed phytochemicals, such as those in dates and other fruits, is based on the combined synergistic and antagonistic actions of the given mixture. Factors that influence these interactions include the bioavailability of the bioactive constituents, interaction with phase 1 and phase 2 metabolic enzymes, inhibition of binding to target proteins, interference with cellular transport processes, and influences onsignalingpathways. Hypothesis non-driven investigations involving "Omic" technologies and other systems biology techniques provide excellent opportunities to facilitate the identification of the biochemical changes and effects of as well as the metabolic influences on dietary phytochemicals [9]. Metabolomic tools, such as high performance liquid chromatography - mass spectrometry and nuclear magnetic resonance spectroscopy, can be used with computational statistics to suggest molecular structures, identify metabolic changes, and quantify effects on health-related biochemical pathways [10]. Metabolomic analysis provides a large data set covering the complex metabolic fingerprints of the dietary mixtures and their effects on cells, animals, or humans. From these data sets biomarkers of phytochemicals and biomarkers of effect can be identified and quantified. The knowledge revealed by the

*Corresponding author: Ghnimi S, Department of Food Science, United Arab Emirates University, UAE, Tel: 00971501389 248; E-mail: Afaf. Kamal-Eldin@uaeu.ac.ae

Received March 01, 2015; Accepted March 03, 2015; Published March 09, 2015

Citation: Ghnimi S, Kamal-Eldin A (2015) Fruit Nutraceuticals Seeking Clinical Evidence and Technological Exploitations. J Bioequiv Availab 7: e64. doi:10.4172/ jbb.10000e64

Copyright: @ 2015 Ghnimi S, et al. This is an open-access article distributed under the terms of the Creative Commons Attribution License, which permits unrestricted use, distribution, and reproduction in any medium, provided the original author and source are credited. 
metabolomic techniques can then be tested and validated by hypothesisdriven experiments that may involve other Omic technologies such as proteomics and transcriptomics. Well-designed experiments may also address interactions between dietary components, e.g. those between phenolic constituents, fiber, and available carbohydrates in the date fruit. Provision of clinical evidence(s) for health benefits will support the exploitation of this abundant food commodity.

Functional foods and nutraceuticals from date fruits provide an opportunity to improve human health, reduce health care costs, and support food security and economic development in producing countries. Date fruits are edible during three developmental stages. At the Khalal stage, dates may be used for jam or dates in-syrup (dibis). Dates at Khalal stage are ideal for meat products, which need the use of antioxidants but not a sweet taste. It would be recommendable blanching unripe date fruits to improve their functional properties due to the inhibition of some enzymes and stabilizing the phenolic compounds and the color, but also enhanced some technological properties such as the water holding capacity and the emulsion stability, which are very important to obtain desirable texture in meat and baked products. Dates at Rutab stage had higher sugar content but a lower phenolic content; therefore, they could be used as natural sweeteners in dairy and pastry products among others. Rutab stage dates may also be used for jam, butter, date bars, and date paste. Tamar stage dates may be processed into date bars, date paste, or date syrup. Date bars fortified with almonds, sesame seeds, oat flakes and skim milk powder can be prepared. These date-based fruit bars can meet the increased demand of international consumers where this fruit is not grown [11]. Because of their low GI and possible anti-diabetic effect and antioxidant properties, date fruits and their syrup, paste, and jelly products could add value to many food matrices.

Date paste and date syrup are two main industrial products produced from date fruits [12]. Replacement of sucrose by date paste or date syrup in different products improves their nutritional value by lowering their GI and increasing their levels of minerals and vitamins. Date paste could be used in the baking industry as a filling in pastries and biscuits, as well as an ingredient in cereals, puddings, breads, cakes, cookies, ice cream, and confectionaries. Most date sugars are invert sugars, which increases the softness of bread and cookies. Date paste has a potential application as a functional ingredient in meat products, e.g. the addition of up to $15 \%$ date paste in the formulation of bologna-type products led to the enhancement of the nutritional (lower fat content and higher fiber content than the control) and technological quality (redder-colored and less hard, chewy and cohesive product) together with a satisfactory sensory quality attributes as compared to controls [13]. Date syrup provides unique functionality when used with milk in producing yogurt, including sweetening, flavoring, and increasing nutritional value. Numerous health benefits beyond nutritional value have been associated with consuming yogurt enriched with up to $10 \%$ date syrup. Date syrup has been used to replace sucrose in yellow and chocolate-flavored layer cakes. Date syrup is also used as a sweetening agent, with a characteristic flavor of mature date fruit, to substitute malt syrup, molasses, glucose syrup, invert sugar, high fructose syrup, and all forms of crystalline sugars.Date juice enriched with pectin and lemon flavoring could be used to prepare date firmer jellies with higher adhesiveness, chewiness, and cohesiveness. The use of date juice in jellies resulted in significantly lesser quantities of sugar, as well as decreased $\mathrm{pH}[13]$.
The incorporation of these potentially functional ingredients obtained from date fruits could be a goodstrategy to enhance the economic importance of this crop in the international trade. Also, with high nutrient value combined with low cost of production, dates have good potential as a fruit alternative in poor regions of the world to help the malnourished to meet their basic energy needs and nutritional requirements. The main challenge for date fruits concerns the assessment of stability and bioavailability of its bioactive substances in food matrices.Some food processing techniques affect the concentrations of nutrients and other bioactive components or their bioavailability. At the same time, advances in food processing technology have provided many techniques for the stabilization of nutrients and other valued substances in food. The use of novel and innovative processing technologies is important for further expansion of dates consumption and marketingas these technologies aim to provide safe, high quality foods (retention of original flavor and color) with desirable nutritional and functional properties [11]. With the possibility of producing date syrups and date pastes with the desired moisture content, texture, and softness, the field to include these basically sweet materials in different foodstuffs is wide open.

\section{References}

1. DeFelice SL (2002) FIM Rationale and Proposed Guidelines for the Nutraceutical Research \& Education Act - NREA. Foundation for Innovation in Medicine.

2. Rahmani AH, Aly SM, Ali H, Babiker AY, Srikar S, et al. (2014) Therapeutic effects of date fruits (Phoenix dactylifera) in the prevention of diseases via modulation of anti-inflammatory, anti-oxidant and anti-tumour activity. Int $\mathrm{J}$ Clin Exp Med 7: 483-491.

3. Baliga MS, Baliga BRV, Kandathil SM, Bhat HP, Vayalil PK (2011) A review of the chemistry and pharmacology of the date fruits (Phoenix dactylifera L.). Food Res Int 44: 1812-1822.

4. Hong YJ, Tomas-Barberan FA, Kader AA, Mitchell AE (2006) The flavonoid glycosides and procyanidin composition of Deglet Noor dates (Phoenix dactylifera). J Agric Food Chem 54: 2405-2411.

5. Hammouda H, Chacrif JK, Trabelsi-Ayadi M, Baron A, Guyot S (2013) Detailed polyphenol and tannin composition and its variability in Tunisian dates (Phoenix dactylifera L.) at different maturity stages. J Agric Food Chem 61: 3252-3263.

6. Alkaabi JM, Al-Dabbagh B, Ahmad S, Saadi HF, Gariballa S, et al. (2011) Glycemic indices of five varieties of dates in healthy and diabetic subjects. Nutr J 10: 59 .

7. Forghani B, Kassaian N, Minaei MT, Zare M, Haghighi S, et al. (2003) Effect of dates (Khorma) on 2 hour postprandial ( $2 \mathrm{hPP}$ ) blood glucose level in type 2 diabetic patients referred to Isfahan Endocrine \& Metabolism Research Center. J ShahidSadoughi University Med. Sci. Health Survey 10: 52-55.

8. Michael HN, Salib JY, Eskander EF (2013) Bioactivity of diosmetin glycosides isolated from the epicarp of date fruits, Phoenix dactylifera, on the biochemical profile of alloxan diabetic male rats. Phytother Res 27: 699-704.

9. Efferth T, Koch E (2011) Complex interactions between phytochemicals. The multi-target therapeutic concept of phytotherapy. Curr Drug Targets 12: 122132.

10. Manach C, Hubert J, Llorach R, Scalbert A (2009) The complex links between dietary phytochemicals and human health deciphered by metabolomics. Mol Nutr Food Res 53: 1303-1315.

11. Siddiq M, Aleid SM, Kader AA (2014) Dates: Postharvest Science, Processing Technology, and Health Benefits. 1st (Edn), John Wiley \& Sons, Hoboken, NJ USA.

12. Kamal-Eldin A, Hashim IB, Mohamed IO (2012) Processing and utilization of palm date fruits for edible applications. Recent Pat Food Nutr Agric 4: 78-86.

13. Manickavasagan A, Mohamed EM, and Sukumar E (2012) Dates: Production, Processing, Food, and Medicinal Values. Medicinal and Aromatic PlantsIndustrial Profiles, CRC Press, Boca Raton, Florida, USA. 